\title{
As intervenções e responsabilidades do enfermeiro de saúde pública em Portugal: uma scoping review
}

\author{
The public health nurse's interventions and responsibilities in Portugal: a scoping review
}

Las intervenciones y responsabilidades del enfermero de salud pública en Portugal: una scoping review

Carmen Cunha'; Andreia Costa"; Maria Adriana Henriques"II

\begin{abstract}
RESUMO
Objetivo: revisar os estudos que referenciam as intervenções e responsabilidades do enfermeiro de saúde pública, desde a criação do Serviço Nacional de Saúde (SNS) Português em 1979, até aos nossos dias. Método: scoping review, seguindo a versão PCC - participants, concept and context, do The Joanna Briggs Institute. Consultamos bases de dados como a Biblioteca do Conhecimento Online, Scientific Eletronic Library Online, EBSCOhost Online Research Databases. O período de publicação foi de 1979 a 2018. Resultados: selecionamos quatro artigos publicados e seis dissertações de mestrado, entre 2010 e 2016 . Os temas têm a ver com atividades e atuações dos enfermeiros, autonomia e competências, fundamentos normativos, perceções de equipas de saúde, transmissão e obtenção de informação em saúde. Conclusão: Os resultados apurados, mesmo que escassos, forneceram pistas para uma reflexão acerca do modo, tipo dos processos de intervenção, destes enfermeiros, que segundo a opinião de alguns deles, compreendem mais as suas competências do que as concretizam.

Descritores: Enfermeiros; saúde pública; competência profissional; revisão.
\end{abstract}

\begin{abstract}
Objective: to review studies that refer to interventions and responsibilities of public health nurses, since the creation of the Portuguese National Health Service (NHS) in 1979, to the present day. Method: scoping review, following the PCC - participants, concept and context version of The Joanna Briggs Institute. We consulted databases such as the Biblioteca do Conhecimento Online, Scientific Electronic Library Online, EBSCOhost Online Research Databases. The publication period was from 1979 to 2018. Results: we selected four published articles and six master's dissertations, between 2010 and 2016. The subjects have to do with nurses' activities and actions, autonomy and competences, normative foundations, perceptions of nursing teams, health, transmission and collection of health information. Conclusion: The results, even if scarce, provided clues for a reflection on the mode, type of intervention processes of these nurses, who, according to some of them, understand their competences more than they concretize them.

Descriptors: Nurses; public health; professional competence; review.

RESUMEN

Objetivo: revisar estudios que se refieren a intervenciones y responsabilidades de enfermeros de salud pública, desde la creación del Servicio Nacional de Salud de Portugal (SNS) en 1979, hasta la actualidad. Método: revisión de alcance, siguiendo el PCC - participantes, concepto y versión de contexto del Instituto Joanna Briggs. Consultamos bases de datos como Biblioteca do Conhecimento Online, Scientific Eletronic Library Online, EBSCOhost Online Research Databases. El período de publicación fue 1979 a 2018. Resultados: seleccionamos cuatro artículos publicados y seis disertaciones de maestría, entre 2010 y 2016. Los temas tienen que ver con las actividades, acciones de los enfermeros, autonomía y competencias, fundamentos normativos, percepciones de los equipos de enfermería, salud, transmisión y recopilación de información sanitaria. Conclusión: los resultados, aunque escasos, proporcionaron pistas para reflexionar sobre el modo, tipo de procesos de intervención de estos enfermeros, quienes, según algunos de ellos, entienden sus competencias más de lo que las concretan.

Descriptores: Enfermeros; salud pública; competencia profesional; revisión.
\end{abstract}

\section{INTRODUÇÃO}

A caracterização de quem é o enfermeiro tem vindo a modificar-se ao longo das décadas. O enfermeiro transformou-se, ao adquirir novos modos de intervenção e responsabilidades, atuação e conhecimentos, e conseguiu um estatuto diferenciado, marcado por uma profissão reconhecida, que se firmou no tempo, onde a qualidade se faz presente.

O enfermeiro é um dos elementos integrantes dos profissionais dos serviços de saúde pública e daí a importância de identificar e discutir as situações em que intervêm, as responsabilidades que assume, bem como as suas competências. Ao referirmo-nos a estas, tomamos como referência o conceito que define competências como: 
[...] Competências acrescidas avançadas: os conhecimentos, habilidades, e atitudes que dão resposta às necessidades, nos diversos domínios de intervenção, acrescentando, às competências de enfermeiro especialista, a perícia fruto da complexidade permanente dos conhecimentos, práticas e contextos numa área de intervenção avançada, potenciando a promoção da qualidade da intervenção do enfermeiro especialista ${ }^{1: 3478}$

E ainda, o:

[...] Enfermeiro especialista é o enfermeiro habilitado com um curso de especialização em enfermagem ou com um curso de estudos superiores especializados em enfermagem, a quem foi atribuído um título profissional que Ihe reconhece competência científica, técnica e humana para prestar, além de cuidados de enfermagem gerais, cuidados de enfermagem especializados na área da sua especialidade ${ }^{2: 2960}$

A enfermagem é uma profissão que exerce o cuidar científico e profissional, centrada em interações. De acordo com a Associação Americana de Saúde Pública, Seç̧ão de Enfermagem de Saúde Pública, enfermagem de saúde pública é a prática de promoção e proteção da saúde das populações, recorrendo aos conhecimentos da enfermagem, social e ciências de saúde pública. ${ }^{3: 3}$

Pretendemos revisar o que existe publicado acerca da intervenção e responsabilidade profissional do enfermeiro de saúde pública em Portugal, face aos desenvolvimentos acontecidos nos últimos anos, caracterizados pela formação especializada e pós-graduada em enfermagem, nomeadamente na enfermagem de saúde pública, pois se nos afigura não estar bem evidenciada.

Ao mencionar intervenção referimo-nos, a um conjunto de ações com um objetivo coerente para provocar mudança ou produzir resultados identificáveis. A responsabilidade profissional do enfermeiro, constitui uma dimensão essencial da profissão de enfermagem, consubstanciando um princípio orientador da atividade dos enfermeiros, como prescreve a alínea a) do no 3 do art. 780 do Estatuto da Ordem dos Enfermeiros, em Portugal ${ }^{4}$.

Persistem questões, como é bem patente no processo da feitura e aprovação da nova Lei de Saúde Pública, espelhos de algum descontentamento dos enfermeiros, na prática dos cuidados de saúde, face a utentes e demais profissionais que com eles os prestam. Estas situações acarretam desconfortos e agravos para o exercício da profissão, pelo que é preciso cada vez mais resgatar, fortalecer e consolidar o nosso campo de atuação.

Tomamos como marco temporal, a criação do Serviço Nacional de Saúde (SNS) português, surgido em 1979, sendo institucionalizado pela Lei no 56/79 de 15 de setembro ${ }^{5}$. O objetivo é revisar os estudos que referenciam as intervenções e responsabilidades do enfermeiro de saúde pública, desde a criação do SNS.

\section{MÉTODO}

A revisão sistemática de literatura (RSL) na modalidade scoping review, apresentada por Arksey e O'Malley ${ }^{6}$ e após sistematizada ${ }^{7}$ foi seguida neste trabalho. A escolha dos estudos não está focada na qualidade da investigação (pois essa avaliação não faz parte, do objetivo da scoping review), envolve ou não recolha de dados, apresenta uma síntese essencialmente qualitativa e raramente quantitativa ${ }^{6}$.

Seguimos os seis passos da scoping review. O protocolo usado é o descrito pelo The Joanna Briggs Institute ${ }^{8}$, e, nas suas etapas, orientou-nos na elaboração da questão de investigação, conduziu-nos na busca bibliográfica, em ordem a obter a melhor informação científica disponível.

A etapa 1 consiste em identificar a questão de investigação a ser respondida, funcionando como um fio condutor, de forma que as estratégias de busca possam ser construídas. Para a pesquisa seguimos a versão PCC - participants, concept e context. Sendo $P$ o tipo de participantes (enfermeiros), $C$ conceito (intervenções, responsabilidades), e $C$ contexto (saúde pública), de acordo com o The Joanna Briggs Institute ${ }^{8}$, com vista a definir os critérios para inclusão e exclusão de estudos na RSL, na modalidade scoping review. Buscamos responder à seguinte questão: $\mathrm{O}$ que tem sido publicado sobre tipos de intervenções / responsabilidades do enfermeiro de saúde pública, desde a criação do SNS, em Portugal.

Na etapa 2 pretendemos identificar os estudos relevantes, que foi feita através da busca nas bases eletrónicas de dados. Consultamos a Biblioteca Virtual da Saúde (BVS), de Portugal; Base de Dados Científicos da Ordem dos Enfermeiros; Repositório Científico de Acesso Aberto de Portugal (RCAAP); Biblioteca do Conhecimento Online (B-ON); PORBASE - Base Nacional de Dados Bibliográficos; Scientific Eletronic Library Online (SciELO); Base de Dados de Enfermagem (BDENF); EBSCOhost Online Research Databases (EBSCO).

Os termos de pesquisa, descritores, usados para as buscas, combinados através de operadores booleanos, foram enfermeiros, intervenções, responsabilidades, saúde, saúde pública, expandidos através dos seus similares ${ }^{9}$. De forma a extrair o máximo de informação, foram combinados assim: enfermeiro OR enfermeiros OR intervenção OR intervenções OR responsabilidades AND saúde OR saúde pública. 
Na etapa 3, seleção dos estudos o processo de revisão consistiu em dois níveis. No primeiro nível, uma revisão através dos títulos e resumos, e no segundo nível, uma revisão do texto total ${ }^{10}$. Nesta, os critérios de inclusão foram: artigos originais de investigação e dissertações de mestrado e doutoramento sobre a realidade portuguesa, ou que abordem os conceitos básicos da questão de investigação (intervenção / intervenções / responsabilidades do enfermeiro e saúde / saúde pública, ou seus similares, competências / papel do enfermeiro, exercício profissional, saúde comunitária), prioritariamente referentes a Portugal, escritos em português, espanhol e inglês. Como critérios de exclusão usamos artigos não originais, que não tivessem a ver com a realidade portuguesa. No final, ficamos com um conjunto de textos, através dos quais fizemos a scoping review.

Para o segundo nível, através da leitura integral dos textos, determinamos se eles cumpriam de facto os critérios de inclusão e de exclusão. A pesquisa foi feita entre março e abril de 2018 por dois investigadores.

A etapa 4, coligir os dados, resultou num texto narrativo tendo por base o ano e tipo de publicação, tipo de estudo, assunto, país e afinidade com o nosso tema.

A etapa 5, sumário dos dados e síntese dos resultados, apresentámo-la em forma narrativa e gráfica, quantificando e apreciando os estudos. Como esta revisão não previu qualquer consulta, a outra entidade, concluímos sem a etapa 6 .

\section{RESULTADOS E DISCUSSÃO}

A pesquisa na B-On, resultou em 2.640 documentos. Refinada através dos limitadores: texto integral, data de publicação - 1979 a 2018, tipos de fontes - revistas académicas, assunto - enfermeiro; nurse; enfermero e idioma português; english; spanish e castilian, reduzimos o total para 124 documentos. Usando o aplicativo Mendeley para proceder à captura destes textos, ele devolveu 44 em pdf. Usado o mesmo protocolo na EBSCO - EBSCOhost Online Research Databases, em concreto na EBSCO Discovery Service, obtivemos 5.614 textos.

A BDENF, acessada através da BVS, não permitiu a utilização dos descritores referidos, mais os respetivos operadores booleanos, pelo que não produziu resultados.

Na SciELO, não foi possível utilizar os operadores e os descritores da mesma forma. Mas obtivemos resultados através dos seguintes descritores e filtros: enfermeiro, mais filtros selecionados - Portugal, Saúde Pública, periódicos, todos os idiomas, áreas temáticas de enfermagem, saúde pública, ambiental e ocupacional na forma de artigo. Apuramos 93 documentos. O aplicativo Mendeley devolveu 62 em pdf. O RCAAP, devolveu apenas 7 artigos.

Para a compilação e seleção dos estudos, realizamos o primeiro nível de seleção, com a leitura dos títulos e resumos dos 106 artigos originais, e selecionamos 11. Destes, apenas 4 dizem respeito a realidades portuguesas, os restantes abordam situações brasileiras, exceto 1, que se situa na Inglaterra. Pelo que reduzimos a quatro os artigos revisados, uma vez que apenas estes atendiam ao último critério, tratar da realidade portuguesa.

Os anos de publicação estendem-se de 1999 a 2017, sendo que o ano de 2016 é o de maior representatividade com 4 artigos. Os temas têm a ver com atividades e atuações dos enfermeiros, autonomia e competências, fundamentos normativos, perceções de equipas de saúde, transmissão e obtenção de informação em saúde. Sobressaem as revistas brasileiras, destacando-se a Revista Brasileira de Enfermagem (REBEn), e a Revista de Enfermagem Referência, portuguesa, com 2 artigos cada.

$\mathrm{Na}$ apresentação gráfica, ilustramos a frequência por anos, dos quatro artigos selecionados, conforme mostra a Figura 1.

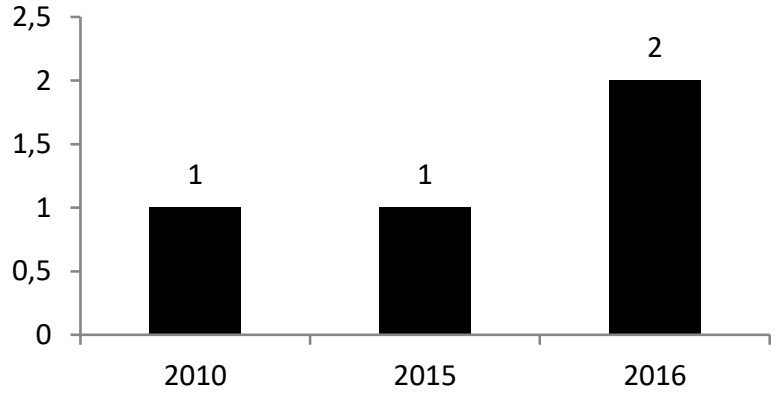

FIGURA 1: Relação ano/número de artigos 
A pesquisa de dissertações e teses, na B-On, resultou em 1355 documentos. Os limitadores texto integral, data de publicação - 1979 a 2018, tipos de fontes - dissertações e teses, assunto - enfermeiro; dissertações de mestrado e intervenções de enfermagem, idioma - português; english; spanish e castilian, reduziu o total para 315 textos.

A aplicação dos critérios de inclusão e exclusão resultou em 6 documentos, relativos a dissertações de mestrado, para leitura. Todas elas dizem respeito a realidades portuguesas, 4 são da Universidade de Lisboa - Escola Superior de Enfermagem de Lisboa (ESEL) e as restantes da Escola Superior de Enfermagem do Porto (ESEP).

As temáticas têm a ver com a intervenção do enfermeiro com adolescentes, intervenções de enfermagem programadas / informática, tecnologia da comunicação em estratégias interventivas, enfermeiro coordenador na unidade de cuidados na comunidade, enfermeiros de cuidados de saúde primários / acessibilidade e proximidade dos cuidados, visibilidade da profissão / enfermeiro e atuação.

Para a apresentação gráfica, relacionamos os dados referentes à data da defesa, conforme apresentado na Figura 2.

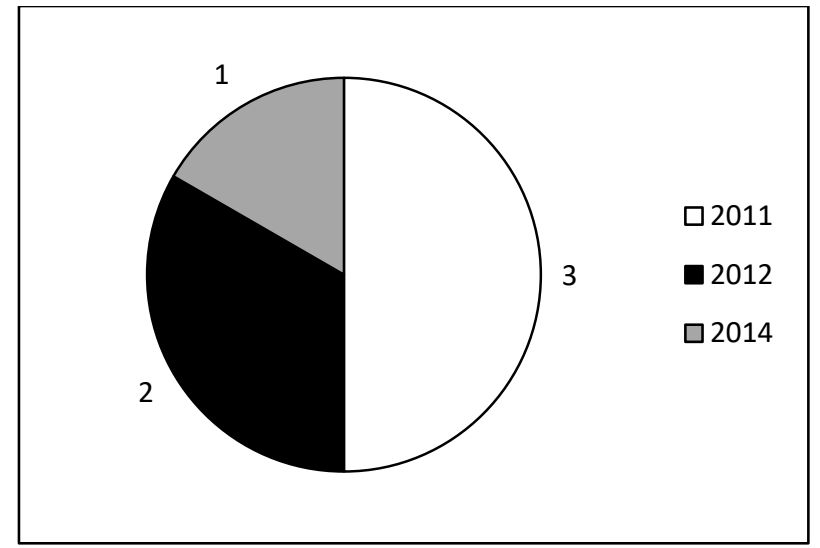

FIGURA 2: Distribuição dissertações/ano

Apresentamos os 4 artigos que selecionamos, na Figura 3.

\begin{tabular}{lll}
\hline Autor(es) & Revista & Título \\
\hline $\begin{array}{l}\text { Guedes VMS, Figueiredo MHS, } \\
\text { Apóstolo, JLA }\end{array}$ & $\begin{array}{l}\text { Revista de Enfermagem } \\
\text { Referência. 2016 jan-fev-mar; } \\
\text { Série IV (8): 27-33 }\end{array}$ & $\begin{array}{l}\text { Competências do Enfermeiro de Cuidados Gerais } \\
\text { em Cuidados de Saúde Primários: da } \\
\text { Compreensão à Concretização }\end{array}$ \\
$\begin{array}{l}\text { Fernandes CS, Martins MM, Gomes } \\
\text { BP, Gomes JA, Gonçalves, LHT }\end{array}$ & $\begin{array}{l}\text { Esc Anna Nery. 2016 20(1):33-7 } \\
\text { Family Nursing Game: Desenvolvendo um jogo de } \\
\text { Temido MAFB, Craveiro I, Dussault G }\end{array}$ & $\begin{array}{l}\text { Revista de Enfermagem } \\
\text { tabuleiro sobre Família }\end{array}$ \\
& $\begin{array}{l}\text { Referência. 2015 jul-ago-set; } \\
\text { Série IV (6): 75-85 }\end{array}$ & $\begin{array}{l}\text { Perceçes de equipas de saúde familiar } \\
\text { portuguesas sobre o alargamento do campo de } \\
\text { exercício da enfermagem }\end{array}$ \\
& Ciência \& Saúde Coletiva. 2010 & Transmissão e obtenção de informação em saúde \\
\hline Rodrigues VMCP & $15(5):$ 2639-46 & \\
\hline
\end{tabular}

FIGURA 3: Relação dos artigos revisados. Período de publicação de 2010 a 2016.

Apresentámo-los, em função dos critérios pré-definidos: enfermeiro / intervenção / responsabilidade / saúde pública / Portugal.

1ㅇ O artigo, Competências do enfermeiro de cuidados gerais em cuidados de saúde primários: da compreensão à concretização ${ }^{11}$, aborda o desenvolvimento de competências compreendidas, e concretizadas pelo enfermeiro de cuidados gerais em cuidados de saúde primários. 
Usa como referencial de competências, o do enfermeiro de cuidados gerais da Ordem dos Enfermeiros ${ }^{12}$, um documento criado com base num modelo ${ }^{13}$, que agrupa 96 competências em três domínios: prática profissional, ética e legal, prestação e gestão dos cuidados e desenvolvimento profissional. Serve-se do conceito de competência, o saber gerir uma situação profissional complexa, influenciada por fatores relativos à história do sujeito, situação profissional e formação ${ }^{14}$.

No geral, as competências são significativamente menos concretizadas do que compreendidas. O que levanta questões, como se os enfermeiros terão oportunidade de pôr em prática os seus conhecimentos ou não. Indagações que se revelam importante para a atuação da enfermagem de saúde pública.

2 $\mathrm{O}$ artigo, Perceções de equipas de saúde familiar portuguesas sobre o alargamento do campo de exercício da enfermagem ${ }^{15}$, tem como temática as perceções de equipas de saúde familiar portuguesas sobre o alargamento do campo de exercício do enfermeiro de Cuidados de Saúde Primários (CSP). A composição da força de trabalho no sistema de saúde português se afigura uma combinação ineficiente de recursos humanos, com uma forte dependência do trabalho médico e um campo de exercício limitado da profissão de enfermagem ${ }^{16}$.

Expandir o campo de exercício do enfermeiro de CSP, a que muitos países recorreram para melhorar o desempenho dos seus sistemas de saúde, pode não ser exequível em Portugal, porque cada país deve encontrar as soluções que melhor se ajustam às suas necessidades em saúde e à oferta de serviços adequada a responder-lhes. Concluem pela existência de um ambiente favorável, a este tipo de mudança na área dos CSP, pois as dinâmicas implementadas estimularam o trabalho em equipa, nas Unidades de Saúde Familiar (USF) ${ }^{16}$.

No entanto, falta unanimidade à atribuição de papéis clínicos mais vastos ao enfermeiro de CSP, pois alguns a veem como desajustada e iníqua. O tema é essencial porque, a discussão sobre o alargamento do campo do exercício da enfermagem, se colocará também no âmbito da saúde pública.

3 $\mathrm{O}$ artigo, Transmissão e obtenção de informação em saúde ${ }^{17}$, cujo conteúdo é identificar de que forma um programa de informação produz alterações no comportamento dos enfermeiros, relativamente à transmissão de informações, e conhecer o grau de obtenção de informações por parte dos doentes.

A comunicação é importante na relação entre o enfermeiro e o doente, e na cooperação e coordenação com todos os elementos da equipe de saúde. Muitas vezes, para além dos doentes e suas famílias, os enfermeiros e a equipa não se sentem bem à vontade para intervir face aos receios, tensão e ansiedade do doente, diante do desconhecido ${ }^{18}$.

Concluiu o autor que seria útil que todas as instituições hospitalares conseguissem implementar nas suas unidades programas de suporte e informação. Sugere que esta comece na formação inicial dos enfermeiros, de modo a contribuir para a melhoria da imagem social da profissão, qualidade dos cuidados prestados ao doente, e humanização dos hospitais e cuidados.

4ㅇ O artigo, "Family nursing game: desenvolvendo um jogo de tabuleiro sobre a família"19, descreve o desenvolvimento de um material didático, jogo de tabuleiro intitulado "Family Nursing Game", construído, testado e validado, para enfermeiros na aprendizagem do conteúdo de enfermagem de família em contexto hospitalar. Afirmam os autores terem optado pelo jogo de tabuleiro, para recriar formas de intervenção e ensinar enfermagem de família. Esta estratégia de intervenção poderá trazer ganhos para todas as suas áreas, incluindo a saúde pública.

Da leitura das 6 dissertações de mestrado destacamos, em função dos mesmos critérios, os aspectos que se seguem.

1a A dissertação, A intervenção do enfermeiro no atendimento a adolescentes no âmbito da consulta de Exame Global de Saúde $(E G S)^{20}$, foi defendida na ESEL. Centra-se nas atividades e estratégias implementadas para desenvolver competências pessoais e profissionais do enfermeiro especialista em saúde infantil.

Refere a autora, partindo do Regulamento dos padrões de qualidade dos cuidados especializados em enfermagem de saúde da criança e do jovem, da Ordem dos Enfermeiros ${ }^{21}$, que se privilegia o método de trabalho por enfermeiro de referência, para uma prestação de cuidados de elevado nível de complexidade.

2a A dissertação, Construção do padrão documental dos cuidados de enfermagem com base em linguagem CIPE: um percurso de desenvolvimento de competências de enfermeiro especialista em saúde infantil e pediatria ${ }^{22}$ foi defendida na ESEL. Centra-se na aplicação informática, que possuiu na sua base de dados um conjunto de intervenções de enfermagem já programadas, e passíveis de serem selecionadas pelos enfermeiros responsáveis pela prestação de cuidados.

3ạ A dissertação, Engagement e suporte social nos enfermeiros de cuidados de saúde primários ${ }^{23}$, foi defendida na ESEP. Aborda o caso dos CSP, onde as alterações organizacionais têm-se vindo a intensificar, para melhorar a satisfação dos utentes, relativamente a este serviço público, em questões de acessibilidade e proximidade dos cuidados. 
4a A dissertação, Enfermeiro coordenador na unidade de cuidados na comunidade: desafios, dificuldades e competências $^{24}$, foi defendida na ESEL. Aborda o processo de reforma dos CSP, caracterizado pela reorganização profunda dos Centros de Saúde (CS), que passam a integrar um Agrupamento de Centros de Saúde, e são definidos como serviços de saúde com autonomia administrativa, constituídos por várias unidades funcionais, que integram um ou mais $\mathrm{CS}^{25}$.

Retiramos reflexões importantes, por se tratar de unidades de cuidados na comunidade, à qual a saúde pública está também intimamente ligada. Ainda o foco nas competências e funções de gestão, que apesar de serem do enfermeiro coordenador da respetiva Unidade de Cuidados na Comunidade (UCC).

5a A dissertação, Contributo da visita domiciliária: perceção dos cuidadores informais dos doentes com demência de Alzheimer ${ }^{26}$, foi defendida na ESEP. Aborda a criação de serviços de aconselhamento, linhas telefónicas de apoio e a utilização das tecnologias de comunicação, que podem constituir estratégias interventivas.

6a A dissertação, Para o percurso de desenvolvimento de competência de enfermeiro ${ }^{27}$, foi defendida na ESEL. Dá visibilidade à profissão, porque desenvolve conhecimentos e habilidades nos enfermeiros que os permite atuar com segurança, baseados na evidência científica, ao destacarem-se como elementos válidos na equipa multidisciplinar.

Embora esta scoping review não tenha fornecido nenhum estudo, em concreto, para ilustrar o nosso propósito, resultou, mesmo assim, em algumas evidências relevantes.

Assim, aparentemente, os enfermeiros compreendem mais as suas competências do que as concretizam; não é seguro, que haja um ambiente favorável à atribuição de mais papéis clínicos aos enfermeiros, ainda que se discuta essa opção no seio das próprias equipas de saúde; que o enfermeiro e a formação em enfermagem, devem investir mais na comunicação / informação, até para melhorar a imagem social da profissão, contribuir para melhores cuidados aos doentes e humanização dos mesmos; a estratégia do uso do jogo na formação e na comunicação poderá ser um dos caminhos a seguir; que o tipo de intervenção esperada do enfermeiro, é a baseada no método de trabalho por enfermeiro de referência; que a experiência do enfermeiro coordenador da UCC, abriu lugar a um novo papel, ainda em construção, para o enfermeiro, e que este tem de mobilizar competências adequadas a estes novos contextos.

Um estudo feito no Brasil também realçou a necessidade do enfermeiro se apoderar do conhecimento para desempenhar funções incentivadoras de ações promotoras de saúde ${ }^{28}$, e uma investigação americana destaca a necessidade de abordagens inovadoras, para tratar questões de competências de enfermagem de saúde pública ${ }^{29}$.

Assim concordamos que, a enfermagem necessita potencializar o status junto à sociedade e, assim, assegurar o reconhecimento amplo e real da profissão ${ }^{30}$.

\section{CONCLUSÃO}

Na sequência desta revisão, é relevante identificar, valorizar e fortalecer, o que é que na prática acrescenta a intervenção e responsabilidades do enfermeiro de saúde pública, em Portugal. Os resultados apurados nos artigos, mesmo que escassos, forneceram pistas para uma reflexão acerca do modo, e tipo dos processos de intervenção, destes enfermeiros, a existência de um ambiente favorável, nas Unidades de Saúde Familiar, à atribuição de mais papéis clínicos ao enfermeiro, a valorização da comunicação, e até mesmo o desenvolvimento de materiais didáticos, como jogos, ainda que segundo a opinião de alguns enfermeiros, compreendam mais as suas competências do que as concretizam.

As dissertações revisadas debruçam-se sobre realidades como, o enfermeiro de referência, o desenvolvimento de competências, ou a experiência do enfermeiro coordenador da Unidade de Cuidados na Comunidade.

Pelo que faz sentido, continuar a busca destas intervenções de enfermagem, e destacar as mais efetivas, para que o decisor político possa optar por elas, numa era de constrangimentos de recursos públicos.

\section{REFERÊNCIAS}

1. Ordem dos Enfermeiros (Pte). Regulamento no 76/2018 de 30 de janeiro 2018. Regulamento da Competência Acrescida Avançada em Gestão. Diário da República, II Série, no 21, 3478-3487. [Internet]. Lisboa, 2018 [cited 2018 May 20]; Available from: https://dre.pt/pesquisa/-/search/114599547/details/normal?l=1

2. Governo Português. Decreto-Lei n. $161 / 96$ de 4 de setembro 1996. Regulamento do exercício profissional dos enfermeiros (REPE). Diário da República, I Série, no 205, 2959- 2962. Lisboa, 1996 [cited 2018 Feb 10]; Available from: https://dre.pt/pesquisa/-/search/241640/details/maximized

3. American Public Health Association, Public Health Nursing Section (EUA). (2013). The definition and practice of public health nursing. [cited $2018 \mathrm{Feb} 15$ ]; Available from:

https://www.apha.org/ /media/files/pdf/membergroups/phn/nursingdefinition.ashx 
4. Assembleia da República (Pt). Lei n. 156/2015, de 16 de setembro de 2015. Altera o Decreto-Lei n.o 104/98 de 21 de abril, que criou a Ordem dos Enfermeiros e aprovou o respetivo Estatuto. Diário da República, I Série, no 181, 8059-8105. Lisboa, 2015 [cited 2018 Feb 10]; Available from: https://dre.pt/home/-/dre/70309896/details/maximized?p_auth=eVIwl6Va

5. Assembleia da República (Pt). Lei no 56/79, de 15 de setembro 1979. Serviço Nacional de Saúde. Diário da República, I Série, no 214, 2357-2363. Lisboa, 1979 [cited 2018 Feb 10]; Available from: https://dre.pt/web/guest/pesquisa//search/369864/details/normal?p_p_auth=JqNc3epD

6. Arksey H, O'Malley L. Scoping studies: towards a methodological framework. International Journal of Social Research Methodology. [cited 2018 Mar 10]; 2005;8(1):19-32. DOI: https://doi.org/10.1080/1364557032000119616

7. Levac D, Colquhoun H, O'Brien KK. Scoping studies: advancing the methodology. Implementation science. 2010 [cited 2018 May 20]; 5(69):1-9. DOI: https://doi.org/10.1186/1748-5908-5-69

8. Peters MDJ, Godfrey CM, McInerney, Soares C B, Khalil H, Parker D. Methodology for JBI scoping reviews. In: The Joanna Briggs Institute Reviewers' Manual. 2015 [cited 2018 May 20]; Available from: http://joannabriggs.org/assets/docs/sumari/ReviewersManual_Methodology-for-JBI-Scoping-Reviews_2015_v2.pdf

9. Wilson R, Godfrey CM, Sears K, Medves J, Ross-White A, Lambert N. Exploring conceptual and theoretical frameworks for nurse practitioner education: a scoping review protocol. JBI Database of Systematic Reviews and Implementation Reports. 2015 [cited 2018 Feb 20]; 13(10):146-55. DOI: https://doi.org/10.11124/jbisrir-2015-2150

10. Jolley RJ, Lorenzetti DL, Manalili K, Lu M, Quan H, Santana MJ. Protocol for a scoping review study to identify and classify patient-centred quality indicators. BMJ Open. 2017 [cited 2018 May 20]; 7(1):1-6. DOI: http://dx.doi.org/10.1136/bmjopen2016-013632

11. Guedes VMS, Figueiredo MHS, Apóstolo JLA. Competencies of general care nurses in primary care: from understanding to implementation. Revista de Enfermagem Referência. 2016 [cited 2018 Feb 5]; V Série (8): 27-33. DOI: http://dx.doi.org/10.12707/RIV15022

12. Ordem dos Enfermeiros (Pt). Competências do enfermeiro de cuidados gerais. [Internet]. Lisboa, 2003 [cited 2018 Apr 6]. Available from: https://www.ordemenfermeiros.pt/arquivo/publicacoes/Documents/divulgar\%20\%20regulamento\%20do\%20perfil_VF.pdf

13. Benner P. De iniciado a perito. Coimbra, Portugal: Quarteto; 2001.

14. Le Boterf G. Compétence et navigation professionelle. Paris, França: Editions d'Organization; 2000.

15. Temido MAFB, Craveiro I, Dussault G. Perceptions of portuguese family health care teams regarding the expansion of nurses' scope of practice. Revista de Enfermagem Referência. 2015 [cited 2018 Apr 12]; IV Série (6): 75-85. DOI: http://dx.doi.org/10.12707/RIV14076

16. Dussault G, Fronteira I. Recursos humanos para a saúde: plano integrado no plano nacional de saúde 2011-2016. Lisboa, Portugal: Alto Comissariado da Saúde; 2010.

17. Rodrigues VMCP. Health transmission and obtaining of information. Ciência \& Saúde Coletiva. 2006 [cited 2018 May 17]; 15(5):2639-46. DOI: http://dx.doi.org/10.1590/\$1413-81232010000500038

18. Levinson P. A arma suave, história natural e futuro da revolução da informação. Lisboa, Portugal: Editorial Bizânico; 1997.

19. Fernandes CS, Martins MM, Gomes BP, Gomes JA, Gonçalves LHT. Family nursing game: developing a board game. Esc. Anna Nery Rev. Enferm. 2016 [cited 2018 Apr 15]; 20(1):33-7. Available from: http://www.scielo.br/scielo.php?pid=S1414$81452016000100033 \&$ script=sci_arttext\&tIng=en

20. Florêncio CAF. A intervenção do enfermeiro no atendimento a adolescentes no âmbito da consulta de Exame Global de Saúde (EGS) [master thesis]. Lisboa, Portugal: Universidade de Lisboa - Escola Superior de Enfermagem; 2014 [cited 2018 Apr 6]. Available from: https://comum.rcaap.pt/handle/10400.26/16310

21. Ordem dos Enfermeiros (Pt). Regulamento dos Padrões de Qualidade dos Cuidados Especializados em Enfermagem de Saúde da Criança e do Jovem. [Internet]. Lisboa, 2011 [cited 2018 Apr 22]. Available from:

https://www.ordemenfermeiros.pt/arquivo/colegios/Documents/MCEESIP_Regulamento_PQCEE\%20_SaudeCriancaJovem.pdf

22. Silva GAGO. Construção do padrão documental dos cuidados de enfermagem com base em linguagem CIPE: um percurso de desenvolvimento de competências para enfermeiro especialista de saúde infantil e pediatria [master thesis]. Lisboa, Portugal: Universidade de Lisboa - Escola Superior de Enfermagem; 2012 [cited 2018 Feb 6]. Available from: http://hdl.handle.net/10400.26/15814

23. Correia VM. Engagement e suporte social nos enfermeiros de cuidados de saúde primários [master thesis]. Porto, Portugal: Universidade do Porto - Escola Superior de Enfermagem do Porto; 2012 [cited 2018 May 16]. Available from: http://hdl.handle.net/10400.26/9318

24. Dias MDFS. Enfermeiro coordenador na unidade de cuidados na comunidade: desafios, dificuldades e competências [master thesis]. Lisboa, Portugal: Universidade de Lisboa - Escola Superior de Enfermagem; 2011 [cited 2018 Mar 10]. Available from: https://comum.rcaap.pt/handle/10400.26/15909

25. Ferreira AL. Contributo da visita domiciliária: perceção dos cuidadores informais dos doentes com demência de Alzheimer [dissertação de mestrado]. Porto, Portugal: Universidade do Porto - Escola Superior de Enfermagem do Porto; 2011 [cited 2018 Jan 8]. Available from: http://hdl.handle.net/10400.26/9516

26. Governo Português. Decreto-Lei no 28/2008 de 22 de fevereiro 2008. Cria os agrupamentos de centros de saúde do Serviço Nacional de Saúde, abreviadamente designados por ACES, e estabelece o seu regime de organização e funcionamento. Diário da República, I Série, no 38, 1182-1189. [Internet]. Lisboa, 2008 [cited 2018 Feb 10]. Available from: https://dre.pt/pesquisa//search/247675/details/maximized 
27. Dias, CED. Para o percurso de desenvolvimento de competência de enfermeiro especialista [dissertação de mestrado]. Lisboa, Portugal: Universidade de Lisboa - Escola Superior de Enfermagem; 2011 [cited 2018 Feb 12]. Available from: http://hdl.handle.net/10400.26/16085

28. Piovesan LR, Schimith MD, Simon BS, Budó MLD, Weiller TH, Brêtas ACP. Health promotion from the perspective of primary health care nurses. Rev. enferm. UERJ. 2016 [cited 2018 Feb 10]; 24(3):e5816,1-6. DOI: http://dx.doi.org/10.12957/reuerj.2016.5816

29. Brown, CL. Linking public health nursing competencies and service-learning in a global setting. Public Health Nursing. 2017 [cited 2018 Feb 22]; 34(5):485-92. DOI: https://doi.org/10.1111/phn.12330

30. Bellaguarda MLR, Padilha MI, Peres MAA, Paim L. The nursing profession: its status - that is the question. Rev. enferm. UERJ. 2016 [cited 2018 Jan 10]; 24(2):e8591,1-5. DOI: http://dx.doi.org/10.12957/reuerj.2016.8591 